\title{
Circulating tumor DNA: a noninvasive biomarker for tracking ovarian cancer
}

\author{
Fang Yang ${ }^{1 \dagger}$, Jun Tang ${ }^{1 \dagger}$, Zihao Zhao ${ }^{1}$, Chunling Zhao ${ }^{1}$ and Yuancai Xiang ${ }^{2 *}$ (D)
}

\begin{abstract}
Ovarian cancer is the fifth leading cause of cancer-related mortality in women worldwide. Despite the development of technologies over decades to improve the diagnosis and treatment of patients with ovarian cancer, the survival rate remains dismal, mainly because most patients are diagnosed at a late stage. Traditional treatment methods and biomarkers such as cancer antigen-125 as a cancer screening tool lack specificity and cannot offer personalized combinatorial therapy schemes. Circulating tumor DNA (ctDNA) is a promising biomarker for ovarian cancer and can be detected using a noninvasive liquid biopsy. A wide variety of ctDNA applications are being elucidated in multiple studies for tracking ovarian carcinoma during diagnostic and prognostic evaluations of patients and are being integrated into clinical trials to evaluate the disease. Furthermore, ctDNA analysis may be used in combination with multiple "omic" techniques to analyze proteins, epigenetics, RNA, nucleosomes, exosomes, and associated immune markers to promote early detection. However, several technical and biological hurdles impede the application of ctDNA analysis. Certain intrinsic features of ctDNA that may enhance its utility as a biomarker are problematic for its detection, including ctDNA lengths, copy number variations, and methylation. Before the development of ctDNA assays for integration in the clinic, such issues are required to be resolved since these assays have substantial potential as a test for cancer screening. This review focuses on studies concerning the potential clinical applications of ctDNA in ovarian cancer diagnosis and discusses our perspective on the clinical research aimed to treat this daunting form of cancer.
\end{abstract}

Keywords: Ovarian cancer, circulating tumor DNA, biomarker, cancer early detection, liquid biopsy

\section{Introduction}

Ovarian cancer is the fifth leading overall cause of cancer-related mortality in women worldwide and the second most common cause of gynecologic cancer-related deaths. Up to $95 \%$ of all ovarian malignancies are diagnosed as epithelial ovarian cancer (EOC) [1]. The most common subtype of EOC is the high-grade serous ovarian cancer (HGSOC) with a prevalence of $52 \%$, followed by endometrioid $(10 \%)$, mucinous $(6 \%)$, and clear cell adenocarcinomas (6\%) [2]. Various efforts have been aimed at treating ovarian carcinoma over the last 30

\footnotetext{
*Correspondence: yuancaix@126.com; yuancaix@swmu.edu.cn

${ }^{\dagger}$ Fang Yang and Jun Tang contributed equally to this work.

${ }^{2}$ Department of Biochemistry and Molecular Biology, Basic Medical College, Southwest Medical University, Luzhou, Sichuan Province, China

Full list of author information is available at the end of the article
}

years, and the disease is curable at an early stage in $90 \%$ of patients [3]. Yet overall disease control remains poor, in part due to the resistance to chemotherapy or other targeted drugs [4-6], which are associated with multiple factors including genome wide mutations [7], epigenetic changes [8], dysfunctionality of DNA repair pathways [9], drug inactivation [10], particular platinum resistance of cancer stem cells (CSCs) [11], and tumor microenvironment [12]. The poor response to this recurrent disease is owing in part to the paucity of effective screening options to discover the specific or typical symptoms at an early stage. Therefore, there is currently an urgent need for optimizing biomarkers that can serve as diagnostic, prognostic, and potential targets for novel therapies that can be used to guide further testing, initiate treatment, and direct the choice of ovarian cancer treatment.

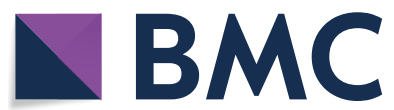

(C) The Author(s) 2021. Open Access This article is licensed under a Creative Commons Attribution 4.0 International License, which permits use, sharing, adaptation, distribution and reproduction in any medium or format, as long as you give appropriate credit to the original author(s) and the source, provide a link to the Creative Commons licence, and indicate if changes were made. The images or other third party material in this article are included in the article's Creative Commons licence, unless indicated otherwise in a credit line to the material. If material is not included in the article's Creative Commons licence and your intended use is not permitted by statutory regulation or exceeds the permitted use, you will need to obtain permission directly from the copyright holder. To view a copy of this licence, visit http://creativecommons.org/licenses/by/4.0/. The Creative Commons Public Domain Dedication waiver (http://creativeco mmons.org/publicdomain/zero/1.0/) applies to the data made available in this article, unless otherwise stated in a credit line to the data. 
Researchers are searching for the dynamic biomarkers applied in non-invasive methods that can indicate the cancer characteristics, such as HE4 [13], transferrin receptor 1 (TFR1) [14], and cancer antigen 125 (CA125) [15]. Among these, CA-125 is the dominantly approved indicator molecule for EOC and the current clinical standard for cancer supervision. Due to the lack of specificity and low sensitivity (50\%-62\%), however, CA-125 levels may be elevated in other conditions such as endometriosis and other malignant tumors, including breast and lung cancers [16]. Of note, nearly $50 \%$ of ovarian neoplasm patients with normal CA-125 levels after chemotherapy showed disease persistence [17]. Thus, CA-125 has limited functionality in the context of asymptomatic women and is not recommended as a screening tool. Recently, some tumor specific DNA, i.e. circulating tumor DNA (ctDNA), was found in patient plasma, and demosntrated high correlation with ovarian cancer prognosis [18-20]. This newly non-invasive biomarker open a new page for ovarian cancer detection and diagnosis. Since the first detection of ctDNA in ovarian cancer in 2012 [21], there has been a significant progress on the role of ctDNA in ovarian cancer and detection methods, especially within the recent five years. Hence, in this review, we summarize the findings of relevant studies of this period. Furthermore, we illustrate the currently available technologies and discuss the existing challenges for analyzing ctDNA in liquid biopsy for application in ovarian cancer.

\section{A brief overview of ctDNA}

ctDNA is emerging as one of the most promising novel alternative prognostic or diagnostic biomarkers for detecting and tracking cancer. The presence of ctDNA in the blood of cancer patients was first reported in the 1970s [22], followed by successful detection of TP53 mutations in body fluids of patients with bladder cancer [23]. During the process of tumor apoptosis, necrosis, or active release, cell-free DNA (cfDNA) is released into the bloodstream. ctDNA is derived from a fraction of total cfDNA [24], and the half-life of ctDNA in the blood circulation is less than $2 \mathrm{~h}$ [25]. ctDNA consists of short DNA fragments (150-200 base pairs). This characteristic, along with its circulating half-life, makes the detection of ctDNA a promising diagnostic tool. A series of comprehensive studies encompassing multiple primary tumor types (such as ovarian, bladder, and colorectal cancers) and/or stages revealed a 6-log variation in ctDNA content $[26,27]$. In addition, ctDNA has been detected in over half the cases of most cancer types [18], and has shown remarkable correlations with the molecular pathology of solid tumors [24, 28, 29]. Moreover, ctDNA may enable the visualization of the whole tumor genome rather than that of a specific section. In addition, ctDNA analysis is noninvasive for obtaining tumor tissue using biopsy and allows serial collection of samples to evaluate the quantitative and compositional changes over time. It is important that ctDNA analysis has demonstrated evolutionary adaptation in response to the inhibitors of platinum chemotherapy and poly ADP ribose polymerase (PARP). For instance, the emergence of bridging reversion mutations result from these treatments in some patients may be easily detected within ctDNA, which offer us an effective tool for cancer supervision [30-32]. Currently, with the development of the analysis and isolation of ctDNA technologies, the noninvasive testing in cancer diagnostic become more widespread [33, 34]. Taken as a whole, ctDNA is gaining momentum as a clinically feasible option capable of reflecting both spatial and temporal tumor heterogeneity $(\mathrm{TH})$.

\section{The detection methods of ctDNA}

In a recent prospective study, gene mutations in ovarian cancer were detected using ctDNA analysis; 94\% (48/51) patients carrying mutation were identified using the blood sample which highly consistent with surgical verification and related to progression-free survival (PFS) [27]. Such high detection efficiency is attributed to the advancement of technologies in genomic analysis. Currently, multiple methods, including polymerase chain reaction (PCR)-based and next generation sequencing (NGS)-based approaches, have been developed to identify the cancer-specific mutations in the bloodstream ctDNA (NGS technologies are summarized in Table 1). PCR-based approaches have been successfully applied in ctDNA analysis; however, they are limited to detection of certain specific known mutations. In fact, a third-generation PCR technology, digital PCR (dPCR) or droplet dPCR (ddPCR), has been shown to possess a high specificity $(81 \%)$ and ultra-sensitivity (99\%) for a known site in ovarian cancer $[26,47,48]$. It allows absolute quantification of nucleic acids and performs target mutant or wild-type analysis of biological samples using fluorescent probes (Fig. 1). In contrast, NGS allows for highly sensitive gene detection against multiple genomic regions in a single assay and has been used for DNA mutation profiling and tumor mutation burden determination [51]. Other approaches, such as whole-genome sequencing (WGS) and cancer-personalized profiling by deep sequencing (CAPP-Seq), which use NGS for the analysis of ctDNA in ovarian cancer, have a broad range of applications, including evaluation of tumor mutation burden [52], detection of epigenetic changes, and diagnostics or identification of resistance mutations [53, 54]. Overall, ctDNA has been detected and analyzed with high 
Table 1 NGS-based methods applied in the detection of ctDNA in ovarian cancer

\begin{tabular}{|c|c|c|c|c|c|c|c|}
\hline Technique & $\begin{array}{l}\text { Targeted or } \\
\text { nontargeted } \\
\text { sequencing }\end{array}$ & $\begin{array}{l}\text { DNA volume of plasma/ } \\
\text { blood }\end{array}$ & $\begin{array}{l}\text { DNA } \\
\text { isolation } \\
\text { (Yes/NO) }\end{array}$ & $\begin{array}{l}\text { Analytical } \\
\text { sensitivity }\end{array}$ & $\begin{array}{l}\text { Quantitative } \\
\text { Results }\end{array}$ & $\begin{array}{l}\text { Type of alterations } \\
\text { detected }\end{array}$ & Ref. \\
\hline AmpliSeq & Targeted sequencing & $2 \mathrm{ml}$ plasma 1-100 ng DNA & Yes & $>2 \%$ & Yes & SNVs, indels & {$[35,36]$} \\
\hline Safe-SeqS & Targeted sequencing & 3 ng DNA & Yes & $0.1 \%$ & Yes & SNVs, indels & {$[37,38]$} \\
\hline TAm-Seq & Targeted sequencing & $<2 \mathrm{ml}$ plasma & Yes & $>2 \%$ & Yes & SNVs, indels & [39] \\
\hline Capp-Seq & Targeted sequencing & 7-32 ng DNA & Yes & $0.02 \%$ & Yes & SNVs, indels & [40] \\
\hline TEC-Seq & Targeted sequencing & 5-250 ng of cfDNA & Yes & $0.05-01 \%$ & Yes & SNVs, indels & {$[41]$} \\
\hline WES & nontargeted sequencing & $50 \mathrm{ng}-1 \mu \mathrm{g}$ DNA & Yes & $>1-3 \%$ & Yes & $\begin{array}{l}\text { SNVs, indels; CNV, rear- } \\
\text { rangements }\end{array}$ & [42-44] \\
\hline WGS & nontargeted sequencing & 250 ng DNA & Yes & $1 \%$ & Yes & $\begin{array}{l}\text { SNVs, indels; CNV, rear- } \\
\text { rangements, chromosomal } \\
\text { aberrations }\end{array}$ & {$[45,46]$} \\
\hline
\end{tabular}

Analytical sensitivity: \% mutant to wild-type abundance ratio; Safe-SeqS Safe-Sequencing System, AmpliSeq Amplicon sequencing, TAm-Seq Tagged-amplicon deep sequencing, CAPP-Seq Cancer Personalized Profiling by deep Sequencing, TEC-Seq Targeted error correction sequencing, CNV copy number variations, SNV single nucleotide variations, WES whole-exome sequencing, WGS whole-genome sequencing.

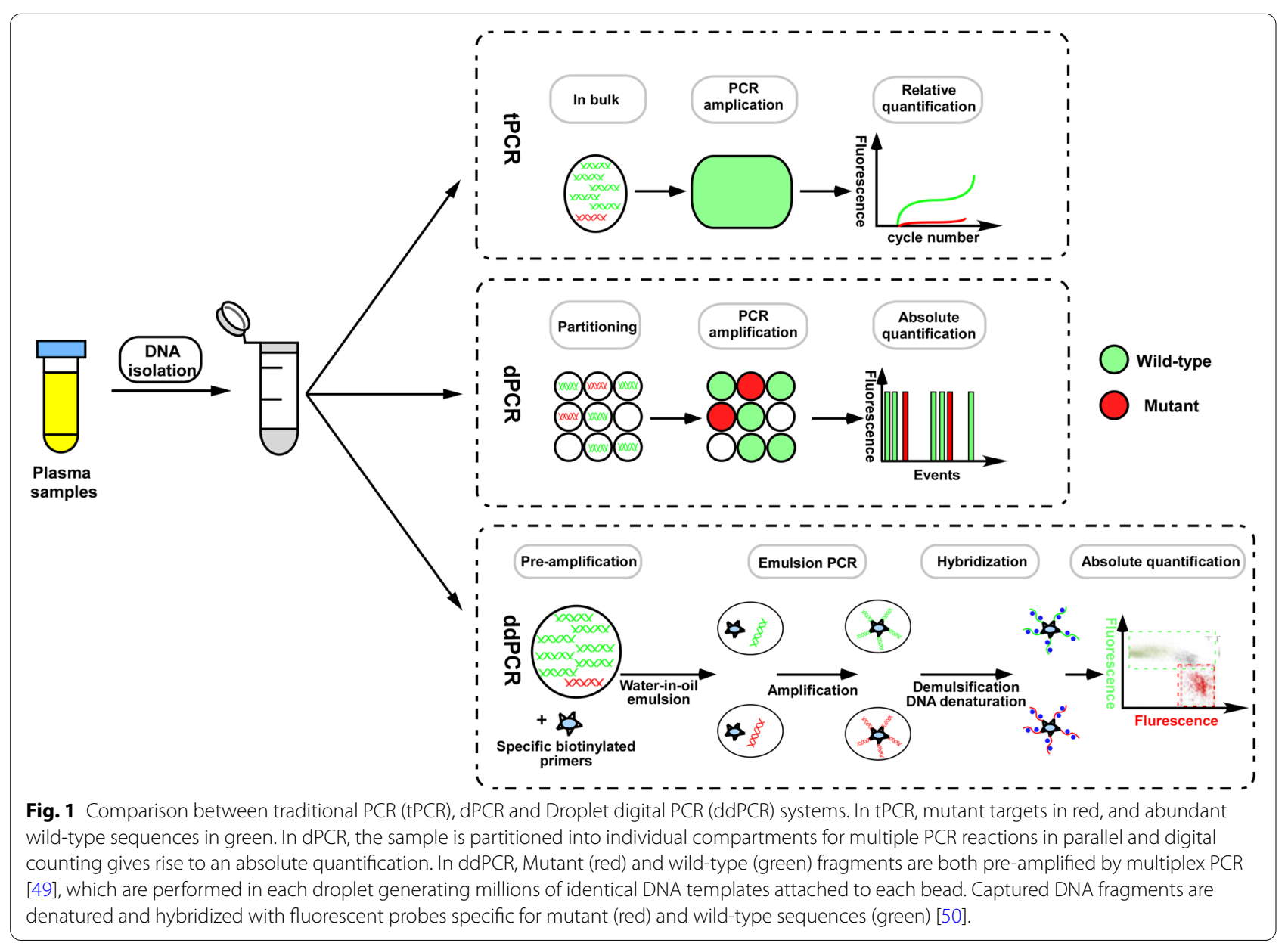

diagnostic sensitivity and specificity in ovarian cancer using a variety of methods (Table 2).

Orthogonal validation has been performed in a limited number of studies to confirm the results obtained.
Interestingly, several studies have shown that the ctDNAbased early detection and/or diagnostic assays may be further advanced by using multi-omics approaches, including those related to proteins, epigenetics, RNA, 


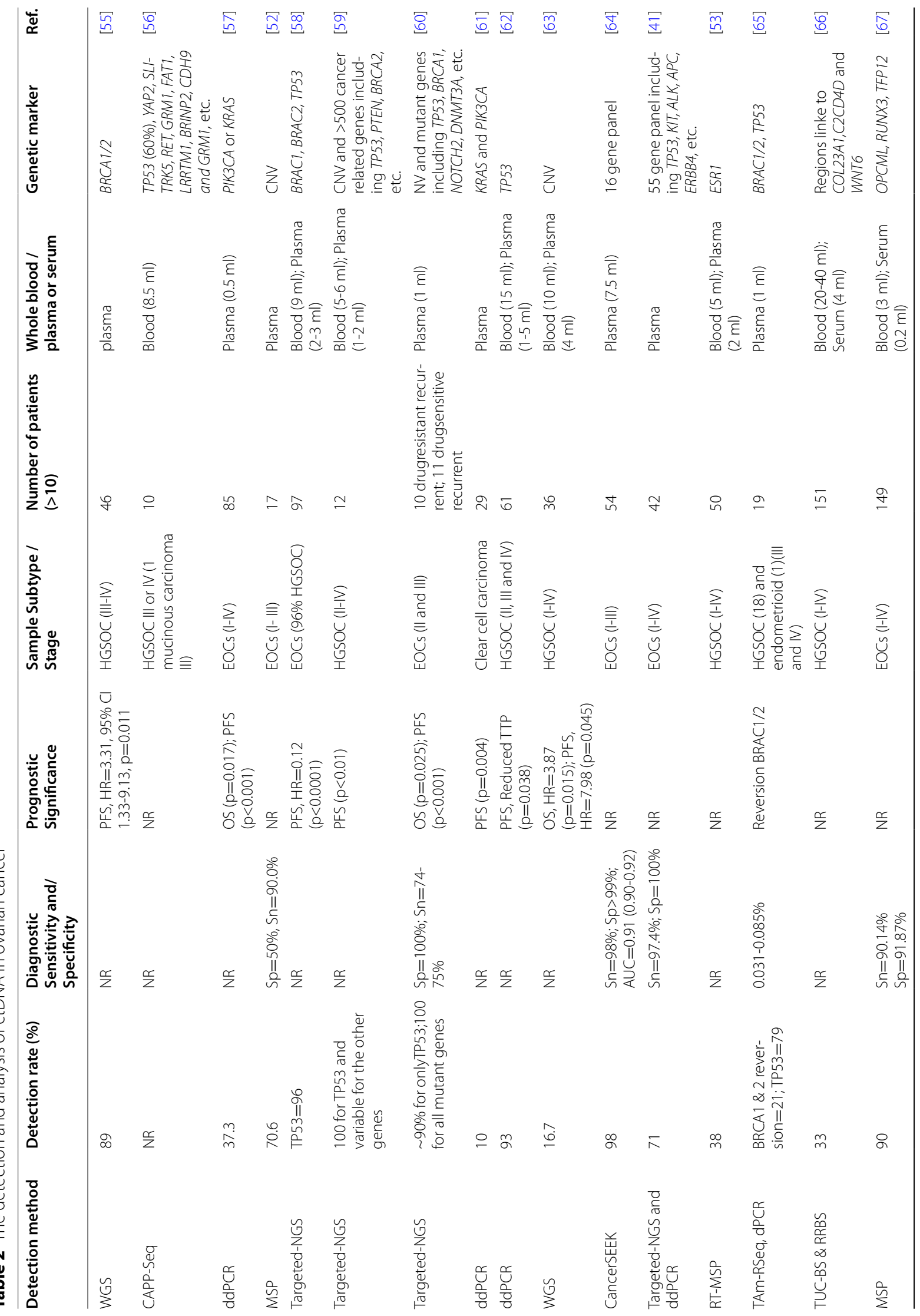




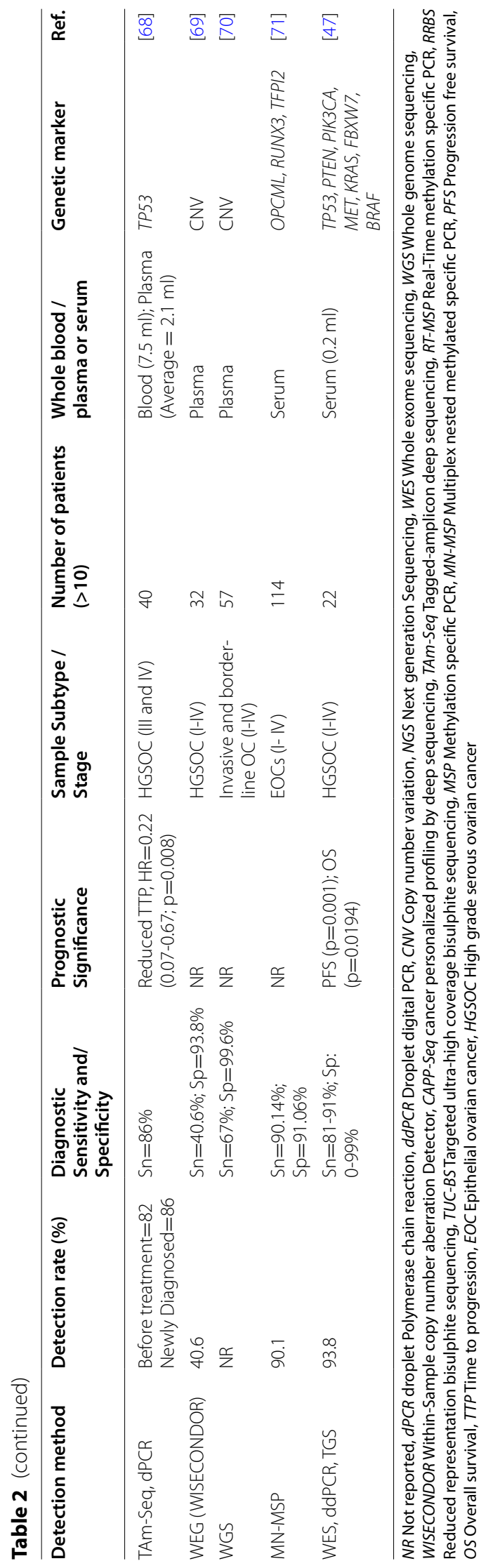


nucleosomes, exosomes, and autoantibodies [72-77]. Many of these strategies may be improved using more permissive tests with greater sensitivity, for instance, by utilizing protein biomarkers, to implement higher diagnostic effectiveness and prognostic accuracy for patients with ovarian cancer.

\section{ctDNA as a screening tool for tracking ovarian carcinoma}

As we all know, the conventional clinical and histological prognostic tools are not accurate for representing the genetic diversity of a tumor, owing to the absence of vital mutational drivers. Moreover, as ovarian cancer is a heterogeneous disease influenced by molecular evolution under treatment exposure [78], it is important to choose a suitable treatment based on the characteristics of genetic and epigenetic processes. Most of the recent developments in ctDNA testing have been directed toward using liquid biopsy. ctDNA has thus emerged as a novel promising non-invasive technology in the diagnosis, prognosis, therapy-response monitoring, resistance emergence, and clonal evaluation of patients with ovarian carcinoma and has been integrated into clinical trials to evaluate disease progression and enable direct treatment [79] (Fig. 2). For example, Phallen et al. [41] and Cohen et al [64] considered the utility of targeted error correction sequencing and the non-invasive blood test CancerSEEK technologies, respectively, to diagnose ovarian cancer and demonstrated that these methods exhibited approximately $97 \%$ sensitivity and $>99 \%$ specificity.

\section{ctDNA is a potential biomarker for the early detection and diagnosis of ovarian carcinoma}

Seventy-five percent of ovarian cancers, especially HGSOC, are diagnosed at an advanced stage, and the survival rates of EOCs have not improved significantly over the past few decades [80]. It is estimated that effective screening could reduce mortality by $10 \%-30 \%$. Therefore, early diagnosis is one of the key requirements in combating this disease.

Even with the advancements in research, the development of ctDNA analysis for the early detection of EOCs demonstrates two major problems: the low abundance of tumor DNA and the high quality of background DNA in blood [81], which affect the sensitivity and reliability of diagnostic results. Thus, to analyze ctDNA in the early stages of cancer, extremely sensitive assays and risk scores for potential mutations are required. To address this point, Zhou et al. [82] suggested an estimated sensitivity of $70 \%$ and specificity of $90 \%$ for quantitative analysis of circulating cfDNA in ovarian cancer. These results imply that this level of specificity of ctDNA analysis is acceptable for ovarian cancer diagnosis, although extensive prospective research is needed to further assess its sensitivity, both independently and in combination with other biomarkers and methods. In addition, Widschwendter et al. [66] analyzed DNA fragments with high quality of background DNA in the bloodstream and demonstrated that abnormal methylation patterns of CpGs can furnish highly specific signals to indicate the presence of cancer. Meanwhile, their results indicate that the methylation patterns in ctDNA may occur prior to diagnosis in a proportion of ovarian cancers and have the potential to guide personalized therapy [66]. Moreover, NGS technology has evolved to achieve reliable sequencing of ctDNA [83], although it has not been routinely implemented in the clinic. Rothwell et al. [84] showed that the ctDNA data obtained by tumor characterisation to guide experimental targeted therapy (TARGET) were in concordance with the matched tumor. Actionable mutations were identified in 41 (out of 100) patients in the study of whom received a corresponding therapy. Cohen et al. [85] reported that by developing CancerSEEK, they were able to achieve early detection rates of $69 \%$ or better in ovarian carcinoma. These results demonstrated that the use of ctDNA could support the selection of patients for early-phase clinical trials.

\section{ctDNA in identification of mutations and characterization of TH in ovarian cancer}

ctDNA extracted from plasma during the course of tumor apoptosis, necrosis, or active release has the potential for transformative applications in ovarian cancer. It is important that ctDNA exists as 150-200 base pairs short fragments that are able to perform PCR-based and NGSbased analyses, thereby probing the mono-mutations within cancer using allele-specific assays. Ogasawara et al. [57] obtained approximately $30 \%$ mutation rates of targeted ctDNA (i.e., KRAS or PIK3CA) from the patients with an ovarian tumor using ddPCR. Of note, Forshew et al .21] identified mutations in the tumor suppressor gene TP53 using Tam-Seq analysis of ctDNA from plasma samples of 46 patients with advanced ovarian cancer and achieved a sensitivity and specificity of $>90 \%$. Beside the detection of instinct mutation of the tumor, ctDNA reflects the dynamic state of targeted gene mutations. For example, two research groups detected reversion mutations of $B R C A 1 / 2$ in ovarian cancer in the process of resistant to the platinum or PARP inhibitors $[32,58]$. These results indicate that this low-cost, highthroughput method may not only facilitate the analysis of ctDNA through a noninvasive "liquid biopsy" for personalized cancer diagnosis but also capture the escape mutations.

Additionally, $\mathrm{TH}$ is another potential issue associated with the treatment of ovarian cancers. Very few research currently have investigated the application of ctDNA 


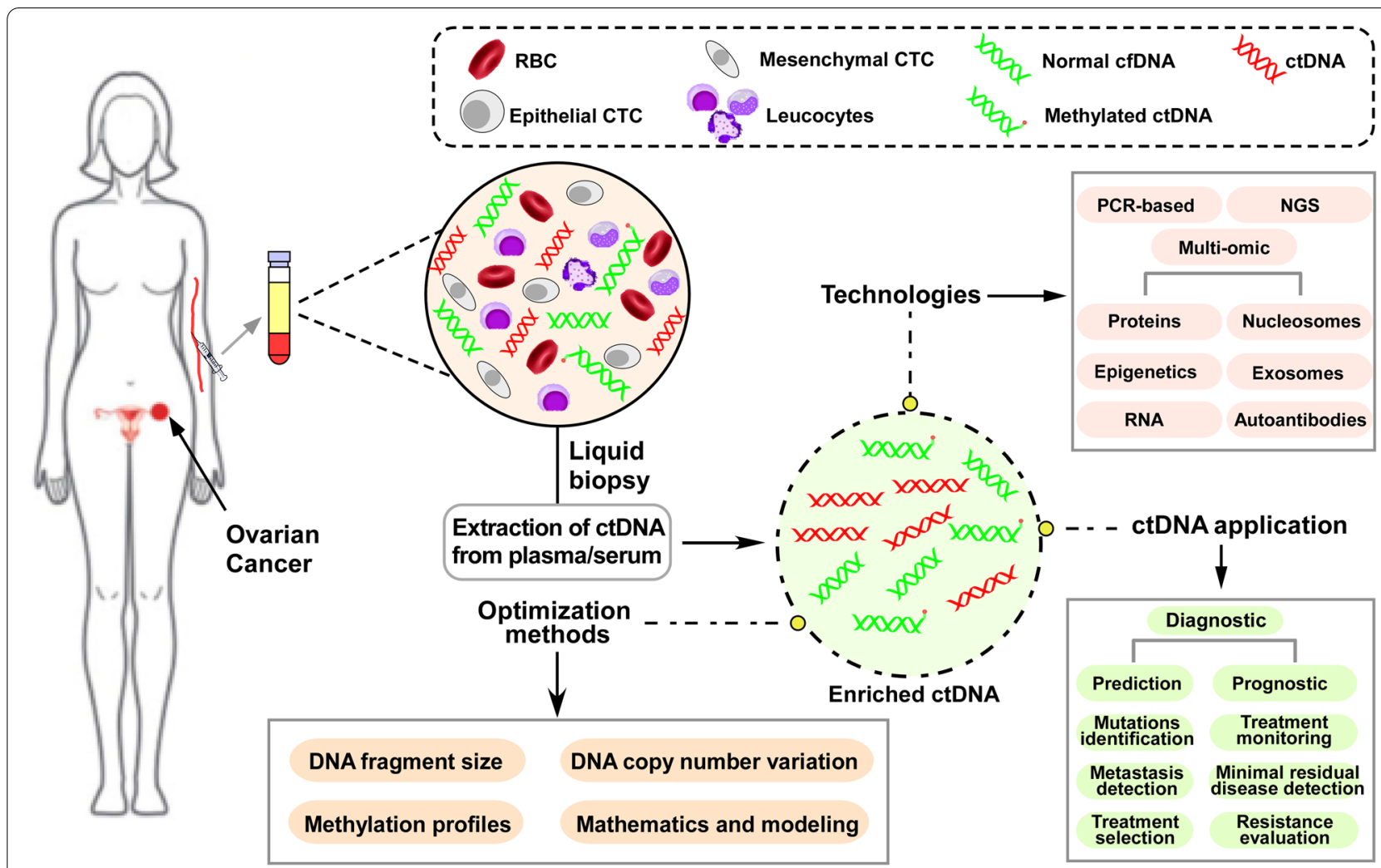

Fig. 2 ctDNA analysis technologies, application and optimization methods in the ovarian cancer patients

analysis in addressing TH. Noguchi et al. [27] recently elucidated the possibility of using ctDNA analysis for supervising treatment responses, including "tumor evolution", which reflects the evolutionary changes in $\mathrm{TH}$ in the $\mathrm{N}$-acetylcysteine-treated patients with advanced ovarian cancer [86]. In addition, Paracchini et al. [55] showed shallow WGS as an inexpensive and useful tool to monitor TH in HGSOC and to obtain an accurate "snapshot" of the tumor genome compared to CA-125, the routine serum biomarker.

\section{Comprehensive profiles of ctDNA to response to treatment in ovarian cancer}

Traditional treatment of ovarian cancer relies on imaging and surgical biopsy. In advanced or recurrent ovarian cancer patients, the former approach only determines the size of the tumor and the extent of changes, and the latter is invasive and sometimes difficult to obtain standard samples. It is well documented that the half-life of the blood protein biomarker CA-125 ranges from 9 to 44 days with the limitations of responding to biological therapies [87-89], implying the seminal advancements in the treatment of ovarian cancer need to continuously explore. A variety of ctDNA quantification methods have several advantages for assessing tumor burden to cure primary and metastatic diseases $[90,91]$. The ctDNA extracted from peripheral blood samples can provide a contemporaneous profile of the tumor genomic landscape. Importantly, ctDNA not only correlates with an early foundation and identification of ovarian cancer but can also be used to monitor treatment efficacy and determine the best treatment method to improve the therapeutic effect [72]. For example, Kim et al. [62] reported that TP53-mut ctDNA demonstrates potential as a tumor-specific biomarker for monitoring treatment response in HGSOC, which is more sensitive than CA-125. Furthermore, a study from Arend et al. group [92] showed that the TP53 variants in ctDNA following neoadjuvant chemotherapy were associated with the treatment response in ovarian cancer patients. Of note, Martignetti et al. [93] identified a rare tumor-specific fibroblast growth factor receptor 2 (FGFR2) fusion gene to monitor the treatment efficacy of advanced ovarian cancer using PCR-based ctDNA, and demonstrated that only the tumor cells with FGFR2 fusion gene ctDNA derived from the patient were sensitive to FGFR2 inhibitor (BGJ398), compared to the cells from other patients. Besides, Noguchi et al. [27] recently reported a series of cancer-specific gene mutations identified via ctDNA from the plasma of 51 pre-treatment patients 
at different stages (I-IV) of ovarian carcinoma. 48 of $51(94 \%)$ patients were found one or more non-synonymous mutations, including HGSOC (TP53, 66.7\%), clear cell carcinoma $(A P C, 30.8 \%)$, endometrioid carcinoma (PIK3CA , 40\%), and mucinous carcinoma (KRAS, 66.7\%). It is worth noting that patients with any such pathogenic mutations demonstrated markedly low PFS ( $p=0.048)$. Overall, the evidence on clinical utility of ctDNA may lead to personalized therapeutic strategies, and guide the clinicians to develop new therapeutic methods to avoid non-effective treatments and improve the outcome of patients.

\section{Prognostication and detection of ovarian minimal residual tumor}

Many tumor patients that undergo curative surgery of their tumor experience recurrence, usually at distant metastatic sites seeded from the primary tumor, and present several mutations. After a surgical resection, it is difficult to distinguish between patients actually achieving remission from those having minimal residual disease. A few studies have demonstrated the ctDNA analysis can prognosticate disease recurrence and minimal residual tumor and may predict progression or response to treatment more rapidly than imaging or CA-125 [57, 94, 95]. Pereira et al. [94] indicated that the utility of personalized ctDNA can identify the presence of residual tumor. They also showed that the predictive lead time associated with the detection of ctDNA is 7 months over that of CT scans. In an exploratory analysis using ctDNA as a biomarker to assess treatment response in ovarian minimal residual tumor, Parkinson et al. [95] demonstrated that ctDNA in patients with relapsed HGSOC correlates with the tumor size at the start of the treatment. They also highlighted that patients with ctDNA levels exhibiting a $>60 \%$ decrease have a remarkably longer time of progression than those with ctDNA levels decreased by $60 \%$ or less after a course of treatment. Arend et al. [92] recently reported that the TP53 variants in ctDNA after neoadjuvant chemotherapy may contribute in determining the presence of minimal residual disease. Additionally, Harris et al. [87] identified plasma ctDNA that permitted the monitoring of cancer patients for relapse and improved therapeutic efficacy via somatic chromosomal rearrangements. Recently, ctDNA from plasma was discovered to be an independent factor for overall survival $(p=0.025)$ and PFS $(p=0.001)$ in patients with recurrent ovarian cancer [60]. These evidence suggest that ctDNA levels are correlated with the recurrence of cancer in patients and highlight the possibility of utilizing the detection of ctDNA mutations as an early indicator of recurrence.

\section{Optimizing ctDNA detection}

The clinical utility of ctDNA in ovarian carcinoma as a diagnostic biomarker has gradually advanced as a minimally-invasive and real-time surrogate for visualizing the development of tumor. However, the advancement in this field of research is relatively recent. In fact, the development of ctDNA analyses was initially impeded by a lack of specific and sensitive techniques for cfDNA quantification. The recent dPCR- or NGS-based assays have advanced critical parameters, such as the mutation allele fraction (MAF) or variant allele fraction (VAF) for the analysis of ctDNA. A large number of ctDNA molecules are expected to possess these two parameters. For example, while the MAF has been reported to be less than $0.1 \%$ in a metastatic setting $[39,96]$, it enables the detection of ctDNA. However, there are two pre-analytical problems associated with optimal ctDNA analysis, including a technical and a biological issue. The technical problem is that many non-designated "mutations" are generated during the progress of analysis. Even in high-throughput screening, the error rate of the compound is higher than that of the available methods. The biological problem is that even with substantial advancement of technologies, the actual samples from plasma or bloodstream (about 5-10 ml) may limit the ctDNA mutation analysis. Hence, the optimization of such technical and biological limitations might lead to new avenues for the clinical applications of this technology.

\section{Potential challenges in the application of ctDNA analysis}

Currently, several potential obstacles exist in the development of a ctDNA-based early tumor diagnostic tool. As noted above, an early detection of tumor is critical in the treatment or morbidity. Therefore, the identification of cancer stage without any prior knowledge of the cancer-specific mutations is challenging and may not be effective in assessing all cancer-associated genes. The cost of ctDNA analysis methods (i.e. NGS) is likely to decrease with further development of the technology. However, even with a reduced cost, the current methods might limit the detection to specific genes or portions of genes, rather than enabling the evaluation of all known genes associated with cancer. Another challenge for early detection is the fact that the cancer may not shed adequate amounts of ctDNA in early-stage disease or during micrometastasis due to the lower disease burden. Therefore, to promote the clinical application of ctDNA analyses, novel approaches for sample collection may be required in the future to stabilize blood cells and/ or reduce the contamination of background DNA in the serum or plasma samples. The third foreseeable hurdle is clonal hematopoiesis, which has not been identified in 
the affected tissue or organ in an occult disease, because of complex ctDNA mutations [97]. Finally, the detection of multiple mutations could offer invaluable information regarding the origin of cancer in a single patient but is not feasible since the infrequency or specificity of ctDNA. At the same time, the mutant ctDNA may display significant false positivity due to non-cancer derived mutations.

\section{Optimization methods}

Although the development of ctDNA analysis is decelerated by some barriers against its clinical utility, certain natural features of ctDNA may strengthen its ability to serve as a diagnostic tool. Plasma cfDNA originating from a neoplasm is considered as ctDNA, and its levels are higher in cancer patients compared to those in healthy individuals [98]. Most studies have mainly focused on genomic alterations for monitoring the tumor and have ignored the changes in ctDNA fragment lengths. Mouliere et al. [99] noted that the size of the DNA fragments (90-150 bp) could be optimized to increase the sensitivity of ctDNA detection. As a result, the median enrichment of ctDNA improved over two-fold in $>95 \%$ of cases. Cristiano et al. [100] further determined that fragmentation profiles may be used to directly detect ctDNA during the early stages of ovarian carcinoma. Another intrinsic feature of ctDNA is the copy number variation $(\mathrm{CNV})$. Because of the small ctDNA fraction derived from early-stage tumors $(<1 \%)$, $\mathrm{CNV}$ presents a hurdle in attaining high-sensitivity testing based solely on a mutational assay. Molparia et al. [101] suggested the potential of CNV detection for ctDNA-based screening as a broad screening methodology. In addition, the CancerSEEK study combined the analysis of specific mutations in ctDNA with a machinelearning algorithm to achieve an accurate diagnosis of ovarian cancer at a sensitivity of $98 \%$ and specificity of $>99 \%$ [64]. Analysis of clonal hematopoiesis mutations in blood with algorithm may also improve the specificity for mutant DNA analysis in ovarian cancer treatment studies. Finally, the methylation profiles may be employed to investigate a possible association between methylation rate of cancer-specific genes and ctDNA [81, 102]. Such methylation patterns in ctDNA can be used to monitor the development of ovarian cancer [52]. Recently, certain mathematical models of ctDNA have been designed for the prediction of tumor volume and to enhance its utility as a tool for monitoring tumor progression. For example, McPherson et al. [103] and Fiala and Diamandis [29] showed that a higher concentration of ctDNA is related to advanced cancer, as well as metastatic cell growth patterns. In addition to ctDNA in blood, ctDNA from ascites correlates with DNA mutations present in tumor biopsies of ovarian cancer. Accordingly, the analysis of fragment size, CNV-based screening, mathematical algorithm models, and ctDNA-associated methylation can facilitate the detection of ctDNA and may provide an alternative approach to the prediction of cancer progression.

\section{Future challenges and prospective}

As described above, ctDNA provides an extremely promising treatment strategy as a noninvasive tool for monitoring ovarian carcinoma during diagnosis and prognosis and while tracking evaluations of patients. In contrast to other traditional biomarkers, such as CA-125, ctDNA demonstrates substantial potential as a key component of ovarian cancer detection trials. Similarly, ctDNA can potentially monitor minimal residual tumor and disease recurrence. Although ctDNA displays a broad prospect in ovarian cancer monitoring, there are several key points that need to be addressed as a priority in the future. First, increase in the accuracy and prevention of the error of ctDNA detection originating from the measurement technique is a critical issue, since a reliable result is key for evaluating the progress of ovarian cancer. Moreover, most ctDNA biomarkers of ovarian cancer currently used in the clinical tests usually detected in other types of cancers as well, leading to possible confusion and interfering with accurate judgment of ctDNA in ovarian cancer supervision, especially in its prediction. Thus, discovering specific ctDNAs originating from ovarian cancer is necessary to improve the detection rate in early screening and to reduce the incidence of this cancer type. Additionally, although several improvement methods mentioned above are being currently employed to optimize the application of ctDNA in ovarian cancer detection, there is a vast scope for advancement. The development of novel methods or combining different high-throughput sequencing and other clinical testing methods to unravel the inherent characteristics of ctDNA is impending and can provide novel insights for into ctDNA-based ovarian cancer detection approaches. More importantly, since ovarian cancer is a heterogeneous disease and ctDNA content can be affected by the tumor stage, heterogeneity, and clonality, the relevance of ctDNA in the clinical treatment of different types, stages, or sizes of ovarian cancer needs to be established in order to provide a clear guidance for cancer monitoring and treatment, which warrant further studies in clinical trials with a significant number of cases. In conclusion, ctDNA acts as a unique biomarker in ovarian cancer management and goes beyond the utilization and assessment of known tumorspecific mutations. Thus, further research is required to establish its clinical utility and ultimately improve personalized or precise treatment of the patients. 


\begin{abstract}
Abbreviations
CA-125: Cancer antigen 125; CNY: Copy number variation (CNV); ctDNA: Circulating tumor DNA; cfDNA: Cell-free DNA; CSCs: Cancer stem cells; dPCR: Digital PCR; ddPCR: Droplet dPCR; EOC: Epithelial ovarian cancer; FGFR2: Fibroblast growth factor receptor 2; HGSOC: High-grade serous ovarian cancer; MAF: Mutation allele fraction; NGS: Next generation sequencing; PARP: Poly ADP ribose polymerase; PCR: Polymerase chain reaction; PFS: Progression-free survival; sWGS: Shallow whole genome sequencing; TARGET: Tumor acARacterisation to Guide Experimental Targeted therapy; TFR1: Transferrin receptor 1; TH: Tumor heterogeneity; VAF: Variant allele fraction; WGS: Whole genome sequencing.
\end{abstract}

\section{Acknowledgements}

We would like to thank Editage (www.editage.cn) for English language editing.

\section{Authors' contributions}

Conceptualization, Y.X; writing—original draft preparation, F. Y, J.T, Z.Z; writing-review and editing, F. Y, C. Z, Y.X; funding acquisition, Y. X and Z.Z. All authors have read and agreed to the published version of the manuscript.

\section{Funding}

This research was funded by Sichuan Science and Technology Program (2019YJ0482), Luzhou City-Southwest Medical University Foundation (2019LZXNYDZ03), Project of Southwest Medical University (2018-ZRQN130), and Program of college student innovation and entrepreneurship (202010632031)

\section{Availability of data and materials}

Not applicable

\section{Declarations}

\section{Ethics approval and consent to participate}

Not applicable

\section{Consent for publication}

Not applicable

\section{Competing interests}

The authors declare no conflict of interest.

\section{Author details}

${ }^{1}$ Department of Physiology, Basic Medical College, Southwest Medical University, Luzhou, Sichuan Province, China. ${ }^{2}$ Department of Biochemistry and Molecular Biology, Basic Medical College, Southwest Medical University, Luzhou, Sichuan Province, China.

Received: 31 May 2021 Accepted: 11 November 2021

Published online: 03 December 2021

\section{References}

1. Lheureux S, Braunstein M, Oza AM. Epithelial ovarian cancer: Evolution of management in the era of precision medicine. CA Cancer J Clin. 2019;69:280-304.

2. Torre LA, Trabert B, DeSantis CE, Miller KD, Samimi G, Runowicz CD, et al. Ovarian cancer statistics, 2018. CA Cancer J Clin. 2018;68:284-96.

3. Peres LC, Cushing-Haugen KL, Köbel M, Harris HR, Berchuck A, Rossing MA, et al. Invasive Epithelial Ovarian Cancer Survival by Histotype and Disease Stage. J Natl Cancer Inst. 2019;111:60-8.

4. Polednak AP. Oropharyngeal Carcinomas Strongly Associated with Human Papillomavirus: Incidence Trends in the Elderly US Population Using SEER vs US Cancer Statistics Databases. J Registry Manag. 2018;45:65-71.

5. Siegel RL, Miller KD, Jemal A. Cancer statistics, 2018. CA Cancer J Clin. 2018;68:7-30

6. Bast RC Jr, Hennessy B, Mills GB. The biology of ovarian cancer: new opportunities for translation. Nat Rev Cancer. 2009;9:415-28.
7. Patch AM, Christie EL, Etemadmoghadam D, Garsed DW, George J, Fereday $S$, et al. Whole-genome characterization of chemoresistant ovarian cancer. Nature. 2015:521:489-94.

8. Chang PY, Liao YP, Wang HC, Chen YC, Huang RL, Wang YC, et al. An epigenetic signature of adhesion molecules predicts poor prognosis of ovarian cancer patients. Oncotarget. 2017;8:53432-49.

9. Barakat BM, Wang QE, Han C, Milum K, Yin DT, Zhao Q, et al. Overexpression of DDB2 enhances the sensitivity of human ovarian cancer cells to cisplatin by augmenting cellular apoptosis. Int J Cancer. 2010;127:977-88.

10. Miyashita H, Nitta Y, Mori S, Kanzaki A, Nakayama K, Terada K, et al. Expression of copper-transporting P-type adenosine triphosphatase (ATP7B) as a chemoresistance marker in human oral squamous cell carcinoma treated with cisplatin. Oral Oncol. 2003;39:157-62.

11. Steg AD, Bevis KS, Katre AA, Ziebarth A, Dobbin ZC, Alvarez RD, et al. Stem cell pathways contribute to clinical chemoresistance in ovarian cancer. Clin Cancer Res. 2012;18:869-81.

12. Senthebane DA, Rowe A, Thomford NE, Shipanga H, Munro D, Mazeedi MAMA, et al. The Role of Tumor Microenvironment in Chemoresistance: To Survive, Keep Your Enemies Closer. Int J Mol Sci. 2017;18:1586.

13. Georgakopoulos P, Mehmood S, Akalin A, Shroyer KR. Immunohistochemical localization of HE4 in benign, borderline, and malignant lesions of the ovary. Int J Gynecol Pathol. 2012;31:517-23.

14. Basuli D, Tesfay L, Deng Z, Paul B, Yamamoto Y, Ning G, et al. Iron addiction: a novel therapeutic target in ovarian cancer. Oncogene. 2017;36:4089-99.

15. Sölétormos G, Duffy MJ, Othman Abu Hassan S, Verheijen RH, Tholander B, Bast RC Jr, et al. Clinical Use of Cancer Biomarkers in Epithelial Ovarian Cancer: Updated Guidelines From the European Group on Tumor Markers. Int J Gynecol Cancer. 2016;26:43-51.

16. Asante DB, Calapre L, Ziman M, Meniawy TM, Gray ES. Liquid biopsy in Ovarian cancer using Circulating Tumor DNA and Cells: Ready for Prime Time? Cancer Lett. 2020;468:59-71.

17. Bast RC Jr. CA 125 and the detection of recurrent ovarian cancer: a reasonably accurate biomarker for a difficult disease. Cancer. 2010;116:2850-3.

18. Ross MG, Russ C, Costello M, Hollinger A, Lennon NJ, Hegarty R, et al. Characterizing and measuring bias in sequence data. Genome Biol. 2013;14:R51.

19. Huang Y, Xu J, Li K, Wang J, Dai Y, Kang Y. A Novel, Personalized DrugScreening System for Platinum-Resistant Ovarian Cancer Patients: A Preliminary Clinical Report. Cancer Manag Res. 2021;13:2849-67.

20. Charo LM, Eskander RN, Okamura R, Patel SP, Nikanjam M, Lanman RB, et al. Clinical implications of plasma circulating tumor DNA in gynecologic cancer patients. Mol Oncol. 2021;15:67-79.

21. Forshew T, Murtaza M, Parkinson C, Gale D, Tsui DW, Kaper F, et al. Noninvasive identification and monitoring of cancer mutations by targeted deep sequencing of plasma DNA. Sci Transl Med. 2012;4:136ra68.

22. Leon SA, Shapiro B, Sklaroff DM, Yaros MJ. Free DNA in the serum of cancer patients and the effect of therapy. Cancer Res. 1997;37:646-50.

23. Sidransky D, Von Eschenbach A, Tsai YC, Jones P, Summerhayes I, Marshall $F$, et al. Identification of p53 gene mutations in bladder cancers and urine samples. Science. 1991;252:706-9.

24. Butler TM, Spellman PT, Gray J. Circulating-tumor DNA as an early detection and diagnostic tool. Curr Opin Genet Dev. 2017:42:14-21.

25. Zhang L, Liang Y, Li S, Zeng F, Meng Y, Chen Z, et al. The interplay of circulating tumor DNA and chromatin modification, therapeutic resistance, and metastasis. Mol Cancer. 2019;18:36.

26. Bettegowda C, Sausen M, Leary RJ, Kinde I, Wang Y, Agrawal N, et al. Detection of circulating tumor DNA in early- and late-stage human malignancies. Sci Transl Med. 2014;6:224ra24

27. Noguchi T, Iwahashi N, Sakai K, Matsuda K, Matsukawa H, Toujima S, et al. Comprehensive Gene Mutation Profiling of Circulating Tumor DNA in Ovarian Cancer: Its Pathological and Prognostic Impact. Cancers (Basel). 2020;12:3382.

28. Wang Y, Li L, Cohen JD, Kinde I, Ptak J, Popoli M, et al. Prognostic Potential of Circulating Tumor DNA Measurement in Postoperative Surveillance of Nonmetastatic Colorectal Cancer. JAMA Oncol. 2019:5:1118-23.

29. Fiala C, Diamandis EP. Utility of circulating tumor DNA in cancer diagnostics with emphasis on early detection. BMC Med. 2018;16:166. 
30. Kondrashova O, Nguyen M, Shield-Artin K, Tinker AV, Teng NNH, Harrell $\mathrm{Ml}$, et al. Secondary Somatic Mutations Restoring RAD51C and RAD51D Associated with Acquired Resistance to the PARP Inhibitor Rucaparib in High-Grade Ovarian Carcinoma. Cancer Discov. 2017;7:984-98.

31. Swisher EM, Lin KK, Oza AM, Scott CL, Giordano H, Sun J, et al. Rucaparib in relapsed, platinum-sensitive high-grade ovarian carcinoma (ARIEL2 Part 1): an international, multicentre, open-label, phase 2 trial. Lancet Oncol. 2017;18:75-87.

32. Christie EL, Fereday S, Doig K, Pattnaik S, Dawson SJ, Bowtell DDL. Reversion of BRCA1/2 germline mutations detected in circulating tumor DNA from patients with high-grade serous ovarian cancer. J Clin Oncol. 2017:35:1274-80.

33. Amant F, Verheecke M, Wlodarska I, Dehaspe L, Brady P, Brison N, et al. Presymptomatic identification of cancers in pregnant women during noninvasive prenatal testing. JAMA Oncol. 2015;1:814-9.

34. Bianchi DW, Chudova D, Sehnert AJ, Bhatt S, Murray K, Prosen TL, et al. Noninvasive Prenatal Testing and Incidental Detection of Occult Maternal Malignancies. JAMA. 2015;314:162-9.

35. Kaisaki PJ, Cutts A, Popitsch N, Camps C, Pentony MM, Wilson G, et al. Targeted Next-Generation Sequencing of Plasma DNA from Cancer Patients: Factors Influencing Consistency with Tumour DNA and Prospective Investigation of Its Utility for Diagnosis. PLoS One. 2016;11:e0162809.

36. Butler KS, Young MY, Li Z, Elespuru RK, Wood SC. Performance characteristics of the AmpliSeq Cancer Hotspot panel v2 in combination with the Ion Torrent Next Generation Sequencing Personal Genome Machine. Regul Toxicol Pharmacol. 2016;74:178-86.

37. Kinde I, Wu J, Papadopoulos N, Kinzler KW, Vogelstein B. Detection and quantification of rare mutations with massively parallel sequencing. Proc Natl Acad Sci U S A. 2011;108:9530-5.

38. I Kinde, C Bettegowd, Y Wang, J Wu, N Agrawal, M le, et al. Evaluation of DNA from the Papanicolaou test to detect ovarian and endometrial cancers. Sci Transl Med. 2013;5:167ra4.

39. Dawson SJ, Tsui DW, Murtaza M, Biggs H, Rueda OM, Chin SF, et al. Analysis of circulating tumor DNA to monitor metastatic breast cancer. N Engl J Med. 2013:368:1199-209.

40. Bratman SV, Newman AM, Alizadeh AA, Diehn M. Potential clinical utility of ultrasensitive circulating tumor DNA detection with CAPP-Seq. Expert Rev Mol Diagn. 2015;15:715-9.

41. Phallen J, Sausen M, Adleff V, Leal A, Hruban C, White J, et al. Direct detection of early-stage cancers using circulating tumor DNA. Sci Transl Med. 2017:9:eaan2415.

42. Klevebring D, Neiman M, Sundling S, Eriksson L, Darai Ramqvist E, Celebioglu F, et al. Evaluation of exome sequencing to estimate tumor burden in plasma. PLoS One. 2014;9:e104417.

43. Warr A, Robert C, Hume D, Archibald A, Deeb N, Watson M. Exome Sequencing: Current and Future Perspectives. G3 (Bethesda). 2015;5:1543-50

44. Manier S, Park J, Capelletti M, Bustoros M, Freeman SS, Ha G, et al. Whole-exome sequencing of cell-free DNA and circulating tumor cells in multiple myeloma. Nat Commun. 2018;91:1691.

45. Chan KC, Jiang P, Zheng YW, Liao GJ, Sun H, Wong J, et al. Cancer genome scanning in plasma: detection of tumor-associated copy number aberrations, single-nucleotide variants, and tumoral heterogeneity by massively parallel sequencing. Clin Chem. 2013;59:211-24.

46. Heitzer E, Ulz P, Belic J, Gutschi S, Quehenberger F, Fischereder K, et al. Tumor-associated copy number changes in the circulation of patients with prostate cancer identified through whole-genome sequencing. Genome Med. 2013;5:30.

47. Pereira E, Camacho-Vanegas O, Anand S, Sebra R, Catalina Camacho S, Garnar-Wortzel L, et al. Personalized circulating tumor DNA biomarkers dynamically predict treatment response and survival in gynecologic cancers. PLoS One. 2015;10:e0145754.

48. Narayan A, Carriero NJ, Gettinger SN, Kluytenaar J, Kozak KR, YockTI, et al. Ultrasensitive measurement of hotspot mutations in tumor DNA in blood using error-suppressed multiplexed deep sequencing. Cancer Res. 2012;72:3492-8.

49. Diaz LA Jr, Bardelli A. Liquid biopsies: genotyping circulating tumor DNA. J Clin Oncol. 2014;32:579-86.

50. Reckamp KL, Melnikova VO, Karlovich C, Sequist LV, Camidge DR, Wakelee $\mathrm{H}$, et al. A highly sensitive and quantitative test platform for detection of NSCLC EGFR mutations in urine and plasma. J Thorac Oncol. 2016;11:1690-700

51. Malapelle U, Pisapia P, Sgariglia R, Vigliar E, Biglietto M, Carlomagno C, et al. Less frequently mutated genes in colorectal cancer: evidences from next-generation sequencing of 653 routine cases. J Clin Pathol. 2016;69:767-71

52. Li S, Huang W, Li Y, Chen B, Li D. A Study of hTERT Promoter Methylation in Circulating Tumour DNAs of Patients with Ovarian Magnificent Tumour. Onco Targets Ther. 2020;13:12317-23.

53. Giannopoulou L, Mastoraki S, Buderath P, Strati A, Pavlakis K, KasimirBauer S, et al. ESR1 methylation in primary tumors and paired circulating tumor DNA of patients with high-grade serous ovarian cancer. Gynecol Oncol. 2018;150:355-60.

54. Liggett TE, Melnikov A, Yi Q, Replogle C, Hu W, Rotmensch J, et al. Distinctive DNA methylation patterns of cell-free plasma DNA in women with malignant ovarian tumors. Gynecol Oncol. 2011;120:113-20.

55. Paracchini L, Beltrame L, Grassi T, Inglesi A, Fruscio R, Landoni F, et al. Genome-wide copy number alterations in circulating tumor DNA as a novel biomarker in high grade serous ovarian cancer patients. Clin Cancer Res. 2020. https://doi.org/10.1158/1078-0432.CCR-20-3345.

56. Noguchi T, Sakai K, Iwahashi N, Matsuda K, Matsukawa H, Yahata T, et al. Changes in the gene mutation profiles of circulating tumor DNA detected using CAPP-Seq in neoadjuvant chemotherapy-treated advanced ovarian cancer. Oncol Lett. 2020;19:2713-20.

57. Ogasawara A, Hihara T, Shintani D, Yabuno A, Ikeda Y, Tai K, et al. Evaluation of Circulating Tumor DNA in Patients with Ovarian Cancer Harboring Somatic PIK3CA or KRAS Mutations. Cancer Res Treat 2020;52:1219-28.

58. Lin KK, Harrell MI, Oza AM, Oaknin A, Ray-Coquard I, Tinker AV, et al. BRCA Reversion Mutations in Circulating Tumor DNA Predict Primary and Acquired Resistance to the PARP Inhibitor Rucaparib in High-Grade Ovarian Carcinoma. Cancer Discov. 2019;9:210-9.

59. Oikkonen J, Zhang K, Salminen L, Schulman I, Lavikka K, Andersson $\mathrm{N}$, et al. Prospective Longitudinal ctDNA Workflow Reveals Clinically Actionable Alterations in Ovarian Cancer. JCO Precis. Oncol. 2019;3:PO.18.00343.

60. Du ZH, Bi FF, Wang L, Yang Q. Next-generation sequencing unravels extensive genetic alteration in recurrent ovarian cancer and unique genetic changes in drug-resistant recurrent ovarian cancer. Mol Genet Genomic Med. 2018;6:638-47.

61. Morikawa A, Hayashi T, Shimizu N, Kobayashi M, Taniue K, Takahashi A, et al. PIK3CA and KRAS mutations in cell free circulating DNA are useful markers for monitoring ovarian clear cell carcinoma. Oncotarget. 2018;9:15266-74.

62. Kim YM, Lee SW, Lee YJ, Lee HY, Lee JE, Choi EK. Prospective study of the efficacy and utility of TP53 mutations in circulating tumor DNA as a non-invasive biomarker of treatment response monitoring in patients with high-grade serous ovarian carcinoma. J Gynecol Oncol. 2019:30:e32.

63. Nakabayashi M, Kawashima A, Yasuhara R, Hayakawa Y, Miyamoto S, lizuka C, et al. Massively parallel sequencing of cell-free DNA in plasma for detecting gynaecological tumour-associated copy number alteration. Sci Rep. 2018:8:11205.

64. Cohen JD, Li L, Wang Y, Thoburn C, Afsari B, Danilova L, et al. Detection and localization of surgically resectable cancers with a multi-analyte blood test. Science. 2018;359:926-30.

65. Weigelt B, Comino-Méndez I, de Bruijn I, Tian L, Meisel JL, García-Murillas I, et al. Diverse BRCA1 and BRCA2 reversion mutations in circulating cell-free DNA of therapy-resistant breast or ovarian cancer. Clin Cancer Res. 2017:23:6708-20.

66. Widschwendter M, Zikan M, Wahl B, Lempiäinen H, Paprotka T, Evans I, et al. The potential of circulating tumor DNA methylation analysis for the early detection and management of ovarian cancer. Genome Med. 2017;9:116.

67. Wang B, Yu L, Luo X, Huang L, Li QS, Shao XS, et al. Detection of OPCML methylation, a possible epigenetic marker, from free serum circulating DNA to improve the diagnosis of early-stage ovarian epithelial cancer. Oncol Lett. 2017;14:217-23.

68. Parkinson CA, Gale D, Piskorz AM, Biggs H, Hodgkin C, Addley H, et al. Exploratory Analysis of TP53 Mutations in Circulating Tumour DNA as Biomarkers of Treatment Response for Patients with Relapsed 
High-Grade Serous Ovarian Carcinoma: A Retrospective Study. PLoS Med. 2016;13:ce1002198.

69. Cohen PA, Flowers N, Tong S, Hannan N, Pertile MD, Hui L. Abnormal plasma DNA profiles in early ovarian cancer using a non-invasive prenatal testing platform: implications for cancer screening. BMC Med. 2016;14:126.

70. Vanderstichele A, Busschaert P, Smeets D, Landolfo C, Van Nieuwenhuysen E, Leunen K, et al. Chromosomal instability in cell-free DNA as a highly specific biomarker for detection of ovarian cancer in women with adnexal masses. Clin Cancer Res. 2017;23:2223-31.

71. Wang B, Yu L, Yang GZ, Luo X, Huang L. Application of multiplex nested methylated specific PCR in early diagnosis of epithelial ovarian cancer. Asian Pac J Cancer Prev. 2015;16:3003-7.

72. Campos-Carrillo A, Weitzel JN, Sahoo P, Rockne R, Mokhnatkin JV, Murtaza M, et al. Circulating tumor DNA as an early cancer detection tool. Pharmacol Ther. 2020;207:107458

73. Chan KC, Jiang P, Chan CW, Sun K, Wong J, Hui EP, et al. Noninvasive detection of cancer-associated genome-wide hypomethylation and copy number aberrations by plasma DNA bisulfite sequencing. Proc Natl Acad Sci U S A. 2013:110:18761-8.

74. Arun G, Diermeier S, Akerman M, Chang KC, Wilkinson JE, Hearn S, et al. Differentiation of mammary tumors and reduction in metastasis upon Malat1 IncRNA loss. Genes Dev. 2016;30:34-51.

75. McAnena P, Brown JA, Kerin MJ. Circulating nucleosomes and nucleosome modifications as biomarkers in cancer. Cancers (Basel). 2017:9:5.

76. Uratani R, Toiyama Y, Kitajima T, Kawamura M, Hiro J, Kobayashi M, et al. Diagnostic potential of cell-free and exosomal MicroRNAs in the identification of patients with high-risk colorectal adenomas. PLoS One. 2016;1 :e0160722

77. Wilson AL, Moffitt LR, Duffield N, Rainczuk A, Jobling TW, Plebanski M, et al. Autoantibodies against HSF1 and CCDC155 as Biomarkers of EarlyStage, High-Grade Serous Ovarian Cancer. Cancer Epidemiol Biomark Prev. 2018;27:183-92.

78. Cheon DJ, Tong Y, Sim MS, Dering J, Berel D, Cui X, et al. A collagenremodeling gene signature regulated by TGF- $\beta$ signaling is associated with metastasis and poor survival in serous ovarian cancer. Clin Cancer Res. 2014;20:711-23.

79. Alves MC, Fonseca FLA, Yamada AMTD, Barros LADR, Lopes A, Silva LCFF, et al. Increased circulating tumor DNA as a noninvasive biomarker of early treatment response in patients with metastatic ovarian carcinoma: a pilot study. Tumour Biol. 2020;42:1010428320919198.

80. Bast RC Jr, Lu Z, Han CY, Lu KH, Anderson KS, Drescher CW, et al. Biomarkers and strategies for early detection of ovarian cancer. Cancer Epidemiol Biomark Prev. 2020;29:2504-12.

81. Sun K, Jiang P, Chan KC, Wong J, Cheng YK, Liang RH, et al. Plasma DNA tissue mapping by genome-wide methylation sequencing for noninvasive prenatal, cancer, and transplantation assessments. Proc Natl Acad SciU S A. 2015;112:E5503-12.

82. Zhou J, Gong G, Tan H, Dai F, Zhu X, Chen Y, et al. Urinary microRNA$30 a-5 p$ is a potential biomarker for ovarian serous adenocarcinoma. Oncol Rep. 2015;33:2915-23.

83. Adalsteinsson VA, Ha G, Freeman SS, Choudhury AD, Stover DG, Parsons $\mathrm{HA}$, et al. Scalable whole-exome sequencing of cell-free DNA reveals high concordance with metastatic tumors. Nat Commun. 2017;8:1324

84. Rothwell DG, Ayub M, Cook N, Thistlethwaite F, Carter L, Dean E, et al. Utility of ctDNA to support patient selection for early phase clinical trials: the TARGET study. Nat Med. 2019;25:738-43.

85. Heintz A, Odicino F, Maisonneuve P, Quinn MA, Benedet JL, Creasman WT, et al. Carcinoma of the Ovary. Int J Gynaecol Obstet. 2006:95:S161-92.

86. McGranahan N, Swanton C. Biological and therapeutic impact of intratumor heterogeneity in cancer evolution. Cancer Cell. 2015;27:15-26.

87. Harris FR, Kovtun IV, Smadbeck J, Multinu F, Jatoi A, Kosari F, et al. Quantification of somatic chromosomal rearrangements in circulating cell-free DNA from ovarian cancers. Sci Rep. 2016;6:29831.

88. Colaković S, Lukiç V, Mitroviç L, Jeliç S, Susnjar S, Marinkoviç J. Prognostic value of CA125 kinetics and half-life in advanced ovarian cancer. Int J Biol Markers. 2000;15:147-52.

89. Bagan P, Berna P, Assouad J, Hupertan V, Le Pimpec BF, Riquet M. Value of cancer antigen 125 for diagnosis of pleural endometriosis in females with recurrent pneumothorax. Eur Respir J. 2008:31:140-2.
90. Abbosh C, Birkbak NJ, Wilson GA, Jamal-Hanjani M, Constantin T, Salari $R$, et al. Phylogenetic ctDNA analysis depicts early-stage lung cancer evolution. Nature. 2017:545:446-51.

91. Diehl F, Schmidt K, Choti MA, Romans K, Goodman S, Li M, et al. Circulating mutant DNA to assess tumor dynamics. Nat Med. 2008; 14:985-90.

92. Arend RC, Londoño Al, Montgomery AM, Smith HJ, Dobbin ZC, Katre $\mathrm{AA}$, et al. Molecular response to neoadjuvant chemotherapy in highgrade serous ovarian carcinoma. Mol Cancer Res. 2018;16:813-24.

93. Martignetti JA, Camacho-Vanegas O, Priedigkeit N, Camacho C, Pereira $\mathrm{E}$, Lin $\mathrm{L}$, et al. Personalized ovarian cancer disease surveillance and detection of candidate therapeutic drug target in circulating tumor DNA. Neoplasia. 2014;16:97-103.

94. Nemtsova MV, Kalinkin Al, Kuznetsova EB, Bure IV, Alekseeva EA, Bykov II, et al. Clinical relevance of somatic mutations in main driver genes detected in gastric cancer patients by next-generation DNA sequencing. Sci Rep. 2020;10:504.

95. Bejar R. Clinical and genetic predictors of prognosis in myelodysplastic syndromes. Haematologica. 2014;99:956-64.

96. Guibert N, Hu Y, Feeney N, Kuang Y, Plagnol V, Jones G, et al. Ampliconbased next-generation sequencing of plasma cell-free DNA for detection of driver and resistance mutations in advanced non-small cell lung cancer. Ann Oncol. 2018;29:1049-55.

97. Silver AJ, Jaiswal S. Clonal hematopoiesis: Pre-cancer PLUS. Adv Cancer Res. 2019;141:85-128.

98. Wan JCM, Massie C, Garcia-Corbacho J, Mouliere F, Brenton JD, Caldas C, et al. Liquid biopsies come of age: towards implementation of circulating tumour DNA. Nat Rev Cancer. 2017;17:223-38.

99. Mouliere F, Chandrananda D, Piskorz AM, Moore EK, Morris J, Ahlborn $L B$, et al. Enhanced detection of circulating tumor DNA by fragment size analysis. Sci Transl Med. 2018:10:eaat4921.

100. Cristiano S, Leal A, Phallen J, Fiksel J, Adleff V, Bruhm DC, et al. Genomewide cell-free DNA fragmentation in patients with cancer. Nature. 2019;570:385-9.

101. Molparia B, Nichani E, Torkamani A. Assessment of circulating copy number variant detection for cancer screening. PLoS One. 2017;12:e0180647.

102. Rusan M, Andersen RF, Jakobsen A, Steffensen KD. Circulating HOXA9methylated tumour DNA: A novel biomarker of response to poly (ADP-ribose) polymerase inhibition in BRCA-mutated epithelial ovarian cancer. Eur J Cancer. 2020;125:121-9.

103. McPherson AW, Roth A, Ha G, Chauve C, Steif A, de Souza CPE, et al. ReMixT: clone-specific genomic structure estimation in cancer. Genome Biol. 2017:18:140.

\section{Publisher's Note}

Springer Nature remains neutral with regard to jurisdictional claims in published maps and institutional affiliations.

$$
\begin{aligned}
& \text { Ready to submit your research? Choose BMC and benefit from: } \\
& \text { - fast, convenient online submission } \\
& \text { - thorough peer review by experienced researchers in your field } \\
& \text { - rapid publication on acceptance } \\
& \text { - support for research data, including large and complex data types } \\
& \text { - gold Open Access which fosters wider collaboration and increased citations } \\
& \text { - maximum visibility for your research: over } 100 \mathrm{M} \text { website views per year }
\end{aligned}
$$

\section{At $\mathrm{BMC}$, research is always in progress.}

Learn more biomedcentral.com/submissions 